\title{
LA LEY DE LA NATURALEZA COMO UNIVERSAL ABSTRACTO. UN ESTUDIO DE LOS PRINCIPIOS MORALES DE JOHN LOCKE \\ A LA LUZ DE SU CRÍTICA A LA IDEA DE SUSTANCIA
}

\author{
THE LAW OF NATURE AS AN ABSTRACT \\ UNIVERSAL. A STUDY OF JOHN LOCKE'S \\ MORAL PRINCIPLES IN LIGHT OF HIS \\ CRITIQUE OF THE IDEA OF SUBSTANCE
}

JoAn Severo Chumbita*

Universidad de Buenos Aires

\begin{abstract}
Resumen: El presente trabajo se propone estudiar la ley de naturaleza a la luz de la crítica que Locke realiza a la idea de sustancia. En este sentido, en primer lugar, se dará cuenta del desplazamiento en la obra de Locke acerca de lo que se entiende por demostración del contenido de la ley de la naturaleza, así como del tipo de prueba exigido. En segundo lugar, se presentarán los diversos contenidos de la ley de la naturaleza que aparecen en el corpus lockeano: los diez mandamientos, el ama a tu prójimo como a ti mismo, el principio de auto-preservación del género humano, la prohibición de acumular frutos para dejar que se pudran, el deber de caridad, de trabajar la tierra y de heredar la propiedad a los hijos. En segundo término, a partir de esta pluralidad de formulaciones, se considerará si resisten la crítica que el propio Locke realiza a la idea
\end{abstract}

*E-mail: joanchumbita@gmail.com 
de sustancia como esencia nominal, esto es, como definición genérica vaga e imperfecta que carece de ideas simples que la sustenten. Por último, se distinguirá esta crítica interna al concepto lockeano de la ley de la naturaleza de la crítica externa en términos de inconsistencia realizada por otros intérpretes.

Palabras Clave: Locke - ley de la naturaleza - esencia nominal - idea de sustancia - idea de Dios.

АвsтRACT: This paper proposes to study the law of nature in light of Locke's critique of the idea of substance. To this end, the first thing which will be accounted for is the displacement in Locke's work regarding what is meant by the demonstration of the content of the law of nature, as well as the type of proof required. In second place, all the different contents of the law of nature that appear in the Lockean corpus will be taken into account: the ten commandments, loving your neighbour as yourself, the principle of self-preservation of the human race, the prohibition stockpiling fruit and leaving it to spoil, the duty of charity, of working the land and of leaving property to children. In third place, the paper will consider whether this plurality of formulations withstands the critique which Locke himself makes of the idea of substance as a nominal essence, that is, as a vague and imperfect generic definition that lacks the simple ideas that would sustain it. Finally, this internal critique of the Lockean concept of the law of nature will be distinguished from the external critique made by other interpreters in terms of inconsistency.

KEYwORDs: Locke - Law of Nature - Nominal essence - Idea of substance - idea of God.

\section{A modo de introducción: la recuperación de un viejo debate}

El problema que supone determinar qué ordena la ley de la naturaleza en la obra de John Locke ha sido tempranamente planteado por Leo Strauss en su polémico libro Natural Right and History. Según Strauss, Locke reduce el contenido de la ley de la naturaleza a lo dictado por la razón allí donde no hay derecho positivo y, en consecuencia, su concepto de ley de la naturaleza poco tiene que ver con la tradición del derecho natural: "arribamos a la conclusión de que Locke no puede reconocer ninguna ley de naturaleza en el sentido propio del término" (Strauss, 1992, 220). A partir de tal hermenéutica, Strauss considera que la concepción lockeana de la ley de la naturaleza resulta en última instancia asimilable a la de Thomas Hobbes (véase Strauss, 1992, 116, 165, 202, 219-227, 245). 
En 1958, John Yolton se ocupó de refutar las formulaciones de Strauss a partir de una reafirmación de la consistencia y la relevancia de la teología cristiana en el corpus lockeano (véase Yolton, 1958, 484-492). A este fin, Yolton redujo la lectura de Strauss a cuatro formulaciones fundamentales: i) la ley de la naturaleza se diferencia de la ley de las Escrituras y es creada por el hombre, no por Dios; ii) la ley de la naturaleza está basada en el derecho natural innato a la felicidad o la auto-preservación, iii) la ley de la naturaleza se reduce a la protección del propio interés: su criterio es el placer y el dolor, la recompensa y el castigo, por lo que tiene una base hedonista; iv) el estado de naturaleza es completamente conflictivo y cada hombre hace lo que quiere (véase Yolton, 1958, 484). Según Yolton, excepto el carácter hedonista de la ley de la naturaleza, todas las demás afirmaciones resultaban explícitamente rechazadas por la obra de Locke (véase Yolton, 1958, 484).

Sin embargo, la crítica de Yolton a la manipulación de las fuentes por parte de Strauss, no resolvía el problema de que la apelación de Locke a las Escrituras exige, según su propia epistemología, de la interpretación de la razón y, por tanto, resulta de por sí crítica y selectiva respecto a sus formulaciones. En esta dirección, no sólo Yolton, sino Raghuveer Singh, Seliger, Gough, así como posteriormente también Richard Ashcraft, Hans Aarsleff y James O. Hancey, coinciden tanto en que la determinación lockeana del contenido de la ley de la naturaleza resulta insuficiente como en que la mediación necesaria de la razón individual para su aplicación práctica conduce, según el propio Locke, a interpretaciones parciales, fanáticas, fantásticas e interesadas en muchos casos (véase Yolton, 1958, 495497; Singh, 1961, 115-116; Martin Seliger, 1963, 345, 354; John Gough, 1964, 18, 34, 43-46; Ashcraft, 1969, 195; Aarsleff, 1969, 134; Hancey, 1976, 442). En efecto, Yolton mismo, al presentar su propia interpretación de los mandatos específicos que, según Locke, componen la ley de la naturaleza, reconoce que constituiría una exageración argumentar que todos los preceptos que Locke señala como contenidos de la ley de la naturaleza se siguen de un principio unitario (véase Yolton, 1958, 487-488). Garotti, en este sentido, advirtió que, según Locke, la ley de la naturaleza no esta ni escrita ni hecha con independencia del trabajo de la razón (véase Garotti, 1961, 156). Aarsleff, por su parte, reconoce también que la ley de la naturaleza carece en el corpus lockeano de una determinación suficiente, pero, en sintonía con la hermenéutica de Yolton y Garotti, rechaza la lectura de Strauss por considerar que esto no necesariamente representa un problema: "lo que Locke quiere decir es que cada hombre, en ausencia de leyes civiles positivas, se guía exclusivamente por su razón en la medida en que él es guiado hacia el orden” (véase Aarsleff, 1969, 133). 
Frente a esta línea interpretativa, Hancey, recupera como propias las críticas de Strauss al concepto lockeano de ley de la naturaleza, sugiriendo que el criterio para dar cuenta de su contenido está dado por la máxima de la salud del pueblo: "Una clara formulación del contenido de la ley de la naturaleza era imposible para Locke. Todo lo que puede decirse sobre ella es lo que Strauss cita como el lema de TT, Salus populi suprema lex esto" (Hancey, 1976, 450). En este sentido, al proponer una formulación tan general, Hancey reconoce que Locke no brinda una "idea clara de un código completo de la ley de la naturaleza” (Hancey, 1976, 450; véase Strauss, 1958, 500). Más recientemente, Simmons, proponiendo una solución intermedia, incorporando las virtudes de ambas líneas interpretativas, la de Strauss y la de Yolton, consideró que, en base al sincretismo existente al interior del pensamiento lockeano, entre teología y secularidad, la ley de la naturaleza, por un lado asume como contenido propio el mandato de la ley revelada (véase Simmons, 1992, 15) y, por el otro, "ley de la naturaleza parece equivaler a poco más que a esto: que hay facultades vinculantes con carácter general, a las que puede apelarse para evaluar y criticar las instrucciones, reglas y acciones humanas" (Simmons, 1992, 103; véase también Abdo Ferez, 2013, 376).

Frente a estas dos líneas interpretativas, una crítica y secular y otra teológica y más contemporizadora, surgirían una serie de interpretaciones planteando un problema de inconsistencia en la formulación lockeana. En este sentido, se parte de la constatación de que tanto en el temprano texto sobre la ley de la naturaleza, Essays on the Law of Nature $(E L N)^{1}$, como en la obra epistemológica más acabada de Locke, An Essay Concerning Human Understanding (Essay) ${ }^{2}$, se sostiene que puede haber un conocimiento demostrativo y formal de la ley de la naturaleza, a pesar de que tal contenido no es presentado en ninguno de los dos textos. A partir de esta falta de demostración del contenido de la ley de la naturaleza, Wolfgang von Leyden objetaría que este problema se sigue del hecho de que existen dos fuentes de obligación moral en la concepción de Locke: una de procedencia divina y otra que surge de la razón humana (véase Leyden, 1964: 52-58). Richard Hall, en cambio, plantearía que la inconsistencia es una consecuencia de que

\footnotetext{
1 Se han traducido todas las citas de esta obra utilizando la edición inglesa de Wolfgang von Leyden (Locke, 1965), consultando la traducción al castellano de Carlos Mellizo (Locke, 2007). Con este mismo criterio, todas las citas de fuentes primarias, así como la bibliografía secundaria para la que no existiera una edición castellana, fueron traducidas por el autor, conservando su referencia al original en inglés.

2 Se empleó en todos los casos la edición inglesa de las obras completas (Locke, 1824, tomo I y II), consultando la traducción de Edmundo O'Gorman.
} 
el conocimiento de la ley moral supone un conocimiento de la esencia real del hombre, el cual no puede alcanzarse según la propia epistemología lockeana (véase Hall, 1981, 38-39, 45-46).

A continuación se recuperará este rico e irresuelto debate en relación con dos cuestiones fundamentales: i) la determinación del contenido mismo de la ley de la naturaleza; ii) la consistencia entre la presentación de Locke del modo en que los hombres acceden a tal contenido y los requisitos establecidos por su epistemología. En este sentido, en primer lugar se dará cuenta del desplazamiento en relación con lo que se entiende por demostración del contenido de la ley de la naturaleza. En segundo lugar, se dará cuenta de los diversos contenidos de la ley de la naturaleza que se formulan en el corpus lockeano: los diez mandamientos, el ama a tu prójimo como a ti mismo, el principio de auto-preservación del género humano, la prohibición de acumular frutos para dejar que se pudran, el deber de caridad, de trabajar la tierra y de heredar la propiedad a los hijos. En tercer lugar, a partir de esta pluralidad de contenidos propuestos, se considerará si resisten la crítica que el propio Locke realiza a la idea de sustancia como esencia nominal, esto es, como definición genérica vaga e imperfecta que carece de ideas simples que la sustenten. Por último, se distinguirá esta crítica interna al concepto lockeano de la ley de la naturaleza de la crítica externa en términos de inconsistencia realizada por otros intérpretes.

\section{El desplazamiento de la exigencia de una demostración formal a la prueba suficiente ofrecida por los milagros del Mesías}

Uno de los rasgos inconfundibles del concepto de ley de la naturaleza de John Locke es su status de mandato divino, no implantado en el hombre, válido como regla de vida universal que debe ser interpretado por parte de la razón individual. En efecto, tanto en los tempranos escritos ELNy Two Tracts on Government $(T G)^{3}$, como en el maduro Essay, así como en los diversos escritos en que aborda la cuestion, The Reasonableness of Christianity $(R)^{4}$, An Examination of $P$. Malebranche's Opinion of Seeing All Things in God $(E M O)^{5}$, Of Ethics in General

3 Se ha utilizado en todos los casos la edición inglesa de Mark Goldie (Locke, 2002).

4 Se ha utilizado en todos los casos la edición inglesa de las obras completas (Locke, 1824, tomo VI), consultando la traducción de Leandro González Puertas (1977).

5 Se recurrió aquí una vez más a la edición de las obras completas (Locke, 1824, tomo VIII), consultando la traducción de José Antonio Robles y Carmen Silva. 
$(E G)^{6}$ y Two Treatises of Government $(T T)^{7}$, Locke considera no sólo que la ley de la naturaleza es un mandato divino, acorde con la naturaleza humana, al cual el hombre no accede de modo innato y que requiere de la interpretación de la razón, sino que tal ley de la naturaleza puede ser demostrada al igual que las verdades matemáticas: "[...] la moralidad es capaz de demostración al igual que las matemáticas. Pues las ideas sobre las que versa la ética, son todas esencias reales, y como tales, imagino, tienen una conexión y un acuerdo descubrible una con otras" (Essay, IV, XII, \$8; véase ELN, II, 132-135; TG, II, 63; $R$, 14-15, 141, 146-147; EMO, 230; EG, \$\$10-12; TT, II, \$\$6-7, 25; Di Biase, 2013: $91,114) .^{8}$

La ley de la naturaleza no puede conocerse a través de la experiencia, en cuanto constituye un deber ser, no un estado del mundo. Su contenido y su demostración, por tanto, serán necesariamente formales, al igual que el contenido y la demostración de las matemáticas (véase Essay, IV, XII, \$8; $R, 139-143$ ). Sin embargo, Locke precisa que los contenidos de la ley de la naturaleza poseen realidad aun cuando carezcan de existencia empírica: "La existencia no es necesaria para hacerlo real [al conocimiento]” (Essay, IV, IV, \$8). La condición de posibilidad del conocimiento es poseer ideas precisas, cuyas ideas complejas se basen en ideas simples y la condición suficiente de la realidad de tales ideas es que respondan a las definiciones previamente estipuladas de determinados arquetipos (Essay, IV, IV, $\$ \$ 1-8 ;$ IV, V, $\$ 11$; IV, XII, $\$ \$ 7,9)$. Esto puede ejemplificarse señalando simplemente que, dada una definición tanto de la unidad como de la adición e igualdad, la suma de dos unidades permitirá hablar de la realidad del conocimiento del número dos como resultante, como igualdad. Lo real, por tanto, no se identifica con lo que posee contenido empírico y lo que se requiere para producirse un conocimiento verdadero de la moral, es decir, de la ley de la naturaleza, es una demostración formal (véase Essay, IV, IV, \$\$6, 8; Essay, II, XXX \$\$1-2; RNB, 255-256; véase Bolton, 1976, 495-496).

6 La edición empleada aquí fue la de Mark Goldie (Locke, 2002), consultando la edición castellana de Blanca Rodríguez López y Diego A. Fernández Peychaux (Locke, 2011).

7 Todas las citas de $T T$, II, se han traducido a partir de la edición inglesa de las obras completas (Locke, 1824, IV), consultando la traducción de Carlos Mellizo (Locke, 1990), así como la edición en inglés de Peter Laslett (Locke, 2004). En el caso de TT, I, se empleó la edición inglesa de las obras completas (Locke, 1824, IV), consultando la traducción de Carmela Gutiérrez de Gambra (Locke, 1966).

8 Para un tratamiento detallado de estas características, así como sobre la continuidad entre los escritos tempranos y tardíos de la obra de Locke sobre esta cuestión, véase Chumbita, 2015. 
Pero si la ley de la naturaleza no requiere de contenido empírico para ser real, sí constituye un problema, para todo objeto de estudio formal, que no se brinde una definición precisa de cada uno de los términos empleados (véase $R N B, 258-259)$. En efecto, si la definición del contenido de la ley de la naturaleza resulta vago y genérico, se vuelve pasible de la crítica que Locke realiza a la idea de sustancia como esencia nominal (véase Essay, IV, XVII, \$\$9-10). En este sentido, la dificultad que presentan los desarrollos de Locke radica en que no abordó en ningún momento la explicitación y derivación del contenido de la ley de la naturaleza de un modo sistemático, sino que, por el contrario, se limitó a dar ejemplos dispersos de tal derivación (Essay, IV, III \$18; véase Hall, 1981, 50, 113; Simmons, 1992, 50; Cox, 1960, 79-81). ${ }^{9}$

En $R$, Locke modifica ligeramente sus afirmaciones de ELN y Essay con respecto al conocimiento demostrativo de la moral. En efecto, si a partir de la posibilidad de demostración formal resulta claro que la ley de la naturaleza puede ser conocida sin necesidad de la revelación positiva de las Escrituras (véase ELN, I, 108-109; Shapiro, 1968, 37, 32, 29; Hancey, 1976, 451), en $R$ Locke sostendrá que la demostración de la ley de la naturaleza sólo fue lograda con la llegada del cristianismo. En efecto, Locke sostiene también aquí que los antiguos podían conocer la ley de la naturaleza, e incluso al Dios único $(R, 135)$. Pero les reprocha el fracaso en su propio terreno por no haber sido capaces de demostrar las verdades morales del mismo modo que se han demostrado las verdades matemáticas $\left(R, 146,143\right.$; véase Hobbes, 1999, XXXI, 311 ${ }^{10}$; Gough, 1964, 7; Hancey, 1976, 443; Abdo Ferez, 2013, 357-358).

No es suficiente que por todas partes estuvieran esparcidos los dichos de los hombres sabios, acorde con la recta razón. La ley de naturaleza es también la ley de la conveniencia: y no es sorprendente que aquellos hombres talentosos y estudiosos de la virtud (que tenían la oportunidad de pensar

Locke presenta como ejemplo la siguiente afirmación: “[ «]Donde no hay propiedad, no hay injusticia[»]", la cual constituye "una proposición tan cierta como cualquier demostración [que se encuentre] en Euclides” o la afirmación de que "«ningún gobierno permite la libertad absoluta»" (Essay, IV, III \$18). En efecto, para Locke la definición de "propiedad» como derecho a y de "justicia» como violación de un derecho, permiten derivar directamente la primera proposición. Lo mismo puede decirse de la segunda a partir de la definición de "gobierno" como establecimiento de reglas a una sociedad, y de "libertad absoluta" como que cada uno haga lo que le plazca (Essay, IV, III \$18).

10 Hobbes ya había hecho este mismo reproche a la tradición filosófica: "ni Platón, ni ningún otro filósofo, hasta ahora ha puesto en orden y ha probado suficientemente, o de manera probable, todos los teoremas de doctrina moral" (Hobbes, 1999, XXXI, 311). 
en una parte específica de ella) llegasen, por la meditación, a iluminar sobre lo justo aun desde la conveniencia observable y la belleza de eso, sin extraer su obligatoriedad de los verdaderos principios de la ley de naturaleza y los fundamentos de la moralidad ( $R, 141-142$; véase también 140).

Además de las críticas a la filosofía por no producir una demostración formal de la moral, Locke sostiene que el cristianismo, a diferencia de la filosofía, ha producido un cuerpo de ética entendido como "principios de la razón" que enseñan "todas los deberes de la vida" $(R, 142)$. En efecto, Locke entiende que el cristianismo ofreció una moral completa y más elevada que la propuesta por la filosofía. Según Locke, el Nuevo Testamento supera a la moral de los "filósofos estudiosos y pensadores", quienes no condenaron el derecho de los padres sobre la vida de los hijos: "ni habían sido capaces de convencer a las partes civilizadas del mundo de que ellos no habían dado, ni podían, sin delito, tomar las vidas de sus hijos" ( $R, 143$; véase también 140; 143-144; TT, II, \$135). Por otra parte, ante el fracaso de la filosofía para producir una demostración formal, Locke reivindicará la prueba ofrecida por el cristianismo, a través de los milagros, para certificar la procedencia divina de sus reglas morales.

No todo escritor de moral o compilador de lo procedente de otros puede por esto ser erigido como un legislador de la humanidad y dictador de reglas que sean válidas porque se hallan en sus libros, bajo la autoridad de éste o aquél filósofo. El que pretenda establecer de este modo sus reglas y hacerlas pasar como lecciones auténticas, deberá mostrar que, o bien construye su doctrina sobre principios de la razón evidentes en sí mismos y de esto deduce todas sus partes, por manifestación clara y evidente, o debe mostrar su comisión del cielo, de que viene con la autoridad de Dios para pronunciar su voluntad y mandamientos al mundo. Del primer modo, nadie que yo sepa, antes de la época de nuestro Salvador, jamás nos dio o estuvo a punto de darnos una moralidad. Es verdad que hay una ley de la naturaleza: pero ¿quién es el que alguna vez la diese o se comprometiese a dárnosla completa como ley, ni más ni menos que lo que estaba contenido en [ella] y tenía la obligación de esa ley? $(R, 142$, véase también 146-147).

Semejante afirmación parece dar por tierra con la clave de lectura que hemos visto presenta Strauss, según la cual la ley de la naturaleza se reduce a una construcción subjetiva de los hombres (véase Strauss, 1992, 116, 165, 202, 219-227, 245). En efecto, como bien destaca Yolton, el contenido de la ley de la naturaleza aparece en esta obra como creación divina y asociado a los mandamientos de las 
Escrituras (véase Yolton, 1958, 484). Al mismo tiempo, si bien Locke seguirá manteniendo que la ley de la naturaleza es demostrable y cognoscible por parte de la razón, aquí la prueba que permite establecer que determinado contenido conforma la ley de la naturaleza está dada por la certificación de la procedencia divina la persona que los enuncia.

\section{La determinación del contenido de la ley de la naturaleza a partir de las máximas del cristianismo}

Establecido este nuevo método para determinar el contenido de los principios morales, Locke señala que la elevación de la moral cristiana está dada por una máxima que Locke entiende muy superior a la ofrecida por todas las demás religiones: el ama a tu prójimo como a ti mismo.

(Lc 10, 25), al abogado que le preguntaba "¿Qué debo hacer para obtener ${ }^{11}$ la vida eterna? Él dijo, ¿Qué está escrito en la ley? ¿Cómo lees?» Él le contestó, Amarás al Señor, tu Dios, con todo tu corazón, y con toda tu alma, y con todas tus fuerzas y con toda tu mente, y al prójimo como a ti mismo. Jesús dijo, «Haz esto y vivirás» ( $\mathrm{R}, 117$; véase también $E G, \$ 11)$.

El cristianismo, según Locke, sostiene el ama a tu prójimo como a ti mismo del Nuevo Testamento pero asume también los diez mandamientos de Moisés (véase también Yolton, 1958, 487-488). Ahora bien, para afirmar que los mandamientos reflejan el contenido de la ley de la naturaleza, como ley universal, Locke considera necesario distinguir estas máximas morales de la ley positiva que Moisés dio a los judíos, tanto en la forma de ley ritual como de ley civil. Se establece así una distinción entre la ley particular de los judíos y la ley moral válida para toda la humanidad: "[...] la parte moral de la ley de Moisés, o la ley moral (que es en todas partes la misma, la regla eterna de justicia), obliga a los cristianos y a todos los hombres, en todas partes, y es para todos los hombres la permanente ley de las obras" $(R, 15)$. Los cristianos no se hallan obligados a observar las leyes positivas o rituales del Antiguo Testamento, pero sí los mandamientos $\left(\mathrm{R}, 13\right.$; véase también $\left.L^{12}, 37 ; \mathrm{R}, 15\right)$.

11 El término en inglés es "inherit", literalmente heredar.

12 A Letter Concerning Toleration. Se utilizó la edición de las obras completas (Locke, 1824, tomo $\mathrm{V})$. 
A estos mandatos divinos, según el Nuevo Testamento, podría ańadirse la desposesión de los bienes externos. Sin embargo, cuando en $R$ Locke refiere al pasaje bíblico en el que Jesús ordena deshacerse de las posesiones terrenales, intenta reducirlo a la ley de la fe (la cual rige exclusivamente para los cristianos, es decir, no como ley universal de la naturaleza), y ordena creer que Cristo es el Mesías. A través de ella, los cristianos pueden acceder a la posibilidad de la gracia divina, y obtener la salvación por esta vía, aun cuando hayan incumplido los mandamientos $(R, 13-15,155)$. En efecto, dada la imposibilidad de sostener al mismo tiempo su teoría de la propiedad ${ }^{13}$ y el mandato divino de desposesión como ley de la naturaleza, Locke pretende considerar este mandato de desposesión como mera prueba de fe y no como un contenido específico de la ley de la naturaleza:

Nuestro Salvador, en respuesta a su pregunta, le dice al joven que para obtener la vida eterna del reino del Mesías debe guardar los mandamientos. Entonces le enumera varios de los preceptos de la ley, [y] el joven dice que los ha observado desde su niñez. Por esto el texto nos lo dice que Jesús lo amaba. Pero nuestro Salvador, para comprobar si de verdad creía que era el Mesías y resolvía tomarlo como su Rey y obedecerle como tal, le manda a dar todo lo que tiene a los pobres y venir y seguirle $\left(R, 119-120^{14}\right)$.

Ahora bien, si en lugar de las formulaciones de $R$ se consideran las de TT, se advierte un nuevo desplazamiento. En efecto, si en $R$ no aparece en ningún

13 Especialmente bajo su forma de apropiación privada, unilateral y desigual. Para un estudio de la relación entre teología y propiedad, véase Chumbita, 2011.

14 Hobbes ya había realizado una interpretación similar del mandato de la desposesión, antes como consejo para obtener la salvación que como contenido de la ley de la naturaleza (véase Hobbes, 1999, XXV, 226). Cabe destacar aquí el problema que presenta la completa ausencia del Nuevo Testamento en TT, II, para la interpretación de Dunn y Waldron, en tanto pretenden derivar consecuencias igualitaristas del cristianismo lockeano (véase Dunn, 1969, 99; Waldron, 2002, 215-216; Waldron, 2005, 498, 506). “"Jesucristo (y San Pablo) puede que no aparezcan en persona en el texto de Two Treatises, pero su presencia no puede pasarse por alto cuando nos encontramos con el carácter normativo de la creación igualitaria de todos los hombres en virtud de su pertenencia a una especie común» [Dunn, 1969, 99]" (Waldron 2005, 498). "He dedicado un capítulo de GLE [2002] a la pregunta acerca de por qué Locke usó tan poco el material del Nuevo Testamento en Two Treatises mientras que utiliza una cantidad considerable de material del Nuevo Testamento en $A$ Letter Concerning Toleration. No tengo nada que agregar aquí [...]. Mi conclusión, si recuerdo bien, era simple: «No sé, no puedo entenderlo» [Waldron, 2002, 215-216]. Creo que decir esto es generalmente mejor que especular sobre una agenda secreta y esotérica" (Waldron, 2005, 506). Waldron parece criticar así las especulaciones de Cox sobre un el secreto hobbesianismo lockeano, en continuidad con las interpretaciones de Strauss (véase Cox, 1960, 71-72). 
momento la idea de que la auto-preservación del género humano constituye el contenido fundamental de la ley de la naturaleza, como en $T T$, tampoco aparece en $T T$ la menor referencia a la ley de la fe o al ama a tu prójimo como a ti mismo sea la máxima suprema de la moral. En TT, II, cuya publicación tiene lugar tan sólo cinco años antes que $R$, y es contemporánea a la de Essay, Locke afirma que el contenido de la ley de la naturaleza se sintetiza en el mandato de auto-preservación del género humano: "El estado de naturaleza tiene una ley de la naturaleza para gobernarlo, la cual obliga a todos: y la razón, que es esa ley, enseña a toda la humanidad, que quiera consultarla ${ }^{15}$, que siendo todos iguales e independientes, ninguno debe lastimar a otro en su vida, salud, libertad o posesiones" (TT, II, $\$ 6$, véase también $\$ \$ 168,149135)$.

A fin de establecer el principio de la auto-preservación del género humano, Locke apela una vez más al status teológico como racional de la ley de la naturaleza (véase Aarsleff, 1969, 133; Hancey, 1976, 450; Simmons, 1992, 103). En efecto, por un lado, la ley de la naturaleza ordena algo que la razón reconoce, la obligación de no dañar a los otros en su propiedad, acorde con su acepción amplia, es decir, como vida, libertad y posesiones (véase $T T$, II $\$ \$ 87,85,171$, 6; $L, 30^{16}$ ). Por otro lado, la auto-preservación del género humano se basa en que la vida humana es propiedad divina, y por ello, junto a las libertades y posesiones, que hacen a su preservación, no debe ser interrumpida por nadie que no sea su creador. "Pues siendo todos los hombres la obra de un Hacedor omnipotente e infinitamente sabio, todos sirvientes ${ }^{17}$ de un amo ${ }^{18}$ soberano, enviado a este mundo por orden suya y para su empresa ${ }^{19}$, ellos son su propiedad, de quien son obra, hechos para durar tanto cuanto sea su gusto y no de otro" (TT, II $\$ 6$; véase también $\$ \$ 7,9,16)$.

Ahora bien, el principio de auto-preservación del género humano supone un orden de prioridades que no resulta fácilmente asimilable al criterio del ama a tu prójimo como a ti mismo, según el cual la vida del prójimo, sea cual fuere su

15 En el original "teaches all mankind, who will but consult it", aquí es importante el matiz introducido por el "who" y el "but", que señalan que si bien la ley enseña a todos, "sin embargo" sólo "quienes" quieran consultarla aprenden lo que enseña.

16 Lo interesante de esta referencia es que se refiere no sólo a las posesiones sino a "the estate", es decir, los bienes inmuebles, los bienes raíces.

17 En el original "servants".

18 En el original "master", término que Locke utiliza para el poseedor de esclavos (véase TT, II, \$85).

19 El término es "business". 
proceder, no se halla nunca subordinada a la propia, ni a la libertad o el derecho sobre las posesiones de nadie. Sin embargo, en $T T$, II la preservación de la propia vida adquiere preeminencia sobre la de los demás:

Cada uno, así como está obligado a preservarse a sí mismo, y a no abandonar su condición ${ }^{20}$ voluntariamente, por la misma razón, cuando su propia preservación no entre en competición, deberá, tanto como pueda, preservar al resto de la humanidad; y no puede, a menos que sea para hacer justicia a un ofensor, tomar o poner en peligro la vida, o lo que tiende a la preservación de su vida, la libertad, la salud, las extremidades o los bienes de otro (TT, II, \$6).

La auto-preservación antepone la vida propia a la de los demás, lo cual se evidencia en la posibilidad, legítima, tanto de aplicar castigos a otros hombres bajo pena capital como de esclavizarlos (véase $T T$, II, $\$ \$ 5,11,16-19,23-24$, 85; Simmons, 1992, 37). ${ }^{21}$ En esta misma dirección, surgen en TT una serie de mandatos divinos vinculados a la teoría de la apropiación, de alcance menos general. En efecto, al mandato divino de la auto-preservación se agrega, como otro ejemplo de contenido de la ley de la naturaleza, el deber de caridad, el cual se define por ser un derecho del necesitado a los medios de subsistencia en caso de extrema necesidad (TT, I, $\$ 42 ; T T$, II, $\$ \$ 5,25-26,183$; ELN, VII, 194-195). Ante la posibilidad de inanición, todos los hombres están obligados a renunciar a una parte de lo que poseen en exceso para evitar la muerte de un semejante (véase Udi, 2012, 165-188, 2014, 149-160; Autor, 2013b, 2014a ${ }^{22}$ ). A estos deberes, puede ańadirse el derecho de herencia, en cuanto Locke también lo presenta como ley de Dios.

[...] el primero y más fuerte deseo que Dios implantón 23 en los hombres, y forjó en los principios mismos de su naturaleza, es aquel de auto-preservación, que es el fundamento de un derecho de las criaturas a su manutención ${ }^{24}$ particular y uso de cada persona individual [para] sí. Pero, junto a esto, Dios

\footnotetext{
20 En el original, "station", que podría traducirse más literalmente como "puesto", refiriendo al estar vivo en este mundo.

21 Para una consideración de cómo el concepto de propiedad privada, en su acepción amplia, como vida, libertad y posesiones, habilita la pena capital por robo, véase Chumbita, 2013a.

22 Para un tratamiento detallado sobre la prohibición de dejar que se pudran los frutos, véase Chumbita, 2011; para una consideración del deber de caridad, véase Chumbita, 2013b; 2014a.

23 En el original "planted".

24 En el original "support".
} 
implantó también en los hombres un fuerte deseo de propagar su especie y continuarse a ellos mismos en su posteridad; y esto da a los hijos un derecho $^{25}$ a participar en la propiedad de sus padres y el derecho a heredar sus posesiones (TT, I, \$88).

Del mismo modo, Locke señala como mandato divino la obligación de no acumular frutos para permitir que se pudran (TT, II, $\$ 31$; véase Chumbita, 2011) y el mandato de trabajar la tierra: "Dios, cuando dio el mundo en común a toda la humanidad, también le ordenó al hombre que trabajara" (TT, II, $\$ 32$; véase también $T T$, I, $\$ 45$, Gén, 3, 19). Todos estos deberes que hemos enumerado hasta aquí se adecúan a la definición de la ley de la naturaleza como mandato divino. A partir de aquí, corresponde someterlos a la crítica que establece la propia epistemología lockeana.

\section{Una consideración del contenido de la ley de la naturaleza a partir de la crítica a la idea de substancia}

En este último apartado se considerará, en efecto, si las diferentes formulaciones que Locke realiza sobre el contenido de la ley de la naturaleza resultan alcanzadas por la crítica que Locke destina a la idea de sustancia como esencia nominal. En este sentido, podemos comenzar recuperando las palabras de Yolton a las que ya hemos hecho referencia en la introducción de este trabajo:

Parecería una burda exageración argumentar que todas estas reglas concretas sean derivables de una ley de la naturaleza, la cual es aprehendida por la función de la razón y el sentido. De hecho, es claramente imposible derivar estos preceptos de ningún principio unitario, ya sea innato, la luz de la razón, o una norma acordada por los hombres (Yolton, 1958, 48826).

La razón por la cual tal derivación de un principio unitario resulta imposible radica no sólo en el hecho de que, como hemos visto en el segundo apartado de este trabajo, Locke no produce una demostración formal al estilo de las matemáticas, sino que, como hemos visto en el tercer apartado, presenta una serie de contenidos que, por su grado de indeterminación y falta de ideas simples que la

25 En el original "title".

26 La lista de Yolton sólo se diferencia de la enumeración aquí presentada en que ella no incorpora el mandato de trabajar la tierra ni hace referencia al mandato de la desposesión (véase Yolton, 1958, 487-488; véase también Abdo Ferez, 2013, 376). 
sustenten, veremos aquí se exponen a la crítica de la idea de sustancia que surge de la propia epistemología lockeana. Para aplicar esta crítica, en primer lugar es preciso presentar la distinción entre esencia real y esencia nominal, para luego abordar la crítica al concepto de sustancia como esencia nominal.

Locke sostiene en Essay que las esencias reales son aquellas que hacen ser a un objeto lo que es (véase Essay, III, III, $\$ 15$; Yolton, 1968, 128-129; Bolton, 1976, 488). Las esencias nominales, por el contrario, se corresponden con la definición por género y especie, la cual no se aplica a los objetos particulares sino a las ideas abstractas de sustancia, tal como, por ejemplo, la definición de hombre en tanto animal racional (véase Essay, III, IV, \$4, 6; Ayers, 1975, 14, 16; Bolton, 1976, 512; Leyden, 1969, 231). La esencia real se caracteriza por

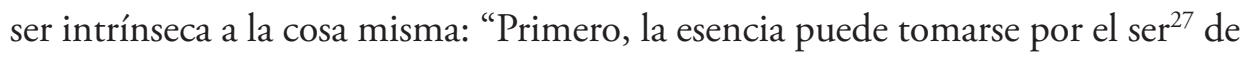
cualquier cosa, por la cual es lo que es. Y así la real, interna pero generalmente, en las sustancias, desconocida constitución de las cosas, de la que dependen sus cualidades descubribles" (Essay, III, III, \$15). La esencia nominal, en cambio, constituye una idea abstracta que los hombres establecemos por convención para distinguir y clasificar los objetos de modo general:

Pero, siendo evidente que las cosas no se ordenan ${ }^{28}$ bajo nombres en clases o especies, sino sólo en cuanto ellas se conforman con ciertas ideas abstractas, a las cuales hemos anexado esos nombres: la esencia de cada género o clase resulta ser nada más que [una] idea abstracta ${ }^{29}$, representada por el nombre general o clasificante ${ }^{30}[\ldots]$. Estas dos clases de esencias, supongo, no estarán impropiamente denominadas, la una real, la otra esencia nominal (Essay, III, III, $\$ 15$, véase también III, VI, $\$ 6$; véase en el mismo sentido $\left.R N B, 256^{31}\right)$.

27 El término es "being".

28 El término es "ranked”, más precisamente "clasifican”; se utilizó la palabra "ordenar" para no repetir "clases" o cambiar el término en este caso.

29 En el original, "comes to be nothing but that abstract idea", también podría traducirse como, "llega a no ser nada sino esa idea abstracta".

30 El término es "sortal".

31 "La inmutabilidad de las esencias [nominales] yace en los mismos sonidos que se supone representan las mismas ideas" (RNB, 256). 
En este pasaje se advierte, a su vez, que Locke no niega la existencia de la esencia real $^{32}$ (véase Yolton, 1968, 127, 133). Por el contrario, se plantea su existencia al interior del objeto, como esencia interna que lo hace ser lo que es, del mismo modo que lo hacía la ontología clásica aristotélica (véase Leyden, 1969, 224-225). La diferencia epistemológica radica en que, según Locke, el entendimiento humano supone la unidad del objeto, pero no puede conocer tal esencia real que hace ser al objeto lo que es, en la medida en que el conocimiento requiere ideas simples, por vía de la sensación o la reflexión, que sirvan de correlato de tal esencia real. Advertimos la unidad del objeto, mas no qué lo hace ser uno, es decir, cuál es su esencia interna: "la sensación nos convence de que hay unas substancias sólidas extensas, y la reflexión de que hay unas [substancias] pensantes” (Essay, II, XXIII, \$29). Sin embargo, advertir la unidad no supone que podamos avanzar con nuestro conocimiento sobre las esencias reales.

[...] las ideas simples que recibimos a través de la sensación y la reflexión son las fronteras ${ }^{33}$ de nuestros pensamientos, más allá de los cuales, la mente, cualquiera sea el esfuerzo que haga, no es capaz de avanzar un ápice; ni puede hacer ningún descubrimiento, cuando intenta entrometerse ${ }^{34}$ en la naturaleza y las causas ocultas de aquellas ideas (Essay, II, XXIII \$29).

De este pasaje se sigue con total claridad tanto que nuestro conocimiento requiere de sustento en ideas simples, como que las esencias reales resultan incognoscibles (véase Essay, IV, VII, \$9). De aquí que, al no acceder a la esencia real de la idea de sustancia, ni a ideas simples de ella, sólo podamos tener su esencia nominal, esto es, una definición por género y especie (véase Leyden, 1969, 226). Esta idea de sustancia es abstracta: "La idea, entonces, que tenemos, [y] a la cual damos el nombre general ["]substancia[»], siendo nada sino el supuesto, pero desconocido soporte de aquellas cualidades que encontramos existentes, de las cuales imaginamos que no pueden subsistir, «sine re substante», sin algo que las sostenga” (Essay, II, XXIII \$2). La esencia nominal define al hombre, por ejemplo, como animal racional, sin un conocimiento de la esencia real del hombre particular ${ }^{35}$ (véase Essay, III, VI, \$\$6, 11; Leyden, 1969, 231). Tal idea

32 Locke critica a la tradición escolástica por haberse extraviado en las discusiones sobre las esencias nominales, aun cuando reconoce que esa tradición no niega la existencia de las esencias reales sino que antes bien las supone (Essay, III, III, \$15).

33 El término es "boundaries", también podría traducirse por "límites".

34 El término es "pry”, también "curiosear", "fisgonear".

35 Esto vale, según Locke, tanto para las sustancias corpóreas como para las espirituales: "Es claro, entonces, que la idea de una substancia corpórea en la materia resulta tan remota para 
de sustancia como esencia nominal, al carecer de ideas simples en las que basarse, resulta por tanto imperfecta e inadecuada desde el punto de vista de la teoría del conocimiento lockeana:

[...] cuáles son las ideas que tenemos de las substancias ya lo mostré arriba. Ahora [veremos que] esas ideas tienen en la mente una doble referencia: 1. Algunas veces están referidas a una supuesta esencia real de cada especie de cosas. 2. Algunas veces solamente están diseñadas para ser imágenes ${ }^{36} \mathrm{o}$ representaciones en la mente de cosas que existen por ideas de esas cualidades que son descubribles en ellas [las cosas]. De ambas maneras, estas copias de aquellos originales y arquetipos son imperfectas e inadecuadas (Essay, II, XXXI \$6).

La idea de sustancia constituye un constructo humano que cumple la función de dar unidad a la percepción, brindando un soporte a los diversos atributos o accidentes que la sensación permite identificar en un objeto dado (véase Yolton, 1968, 133-134, 141-142; Bolton, 1976, 491). Pero en cuanto carece de ideas simples, no es sino un universal abstracto que no provee conocimiento más allá de ciertas proposiciones vagas e imperfectas (véase Essay, II, XXIII, $\$ \$ 2,4$, IV, VI, $\$ \$ 7,11$; Hall, 1981, 71, 74-76; Abdo Ferez, 2013, 346, 351-354). En el caso de los cuerpos "nuestra investigación sobre el conocimiento de las substancias", debido a la falta de ideas adecuadas, no nos permite "ir más allá de donde nos conducen las ideas simples de nuestra esencia nominal, lo cual es muy poco más allá de sí mismas, y en consecuencia nos ofrecen muy escasas verdades ciertas, universales y útiles" (Essay, IV, XII, \$9, véase también $\$ 8, I V$, XVII, \$9). De este modo, más allá de la controversia por la ambigüedad con que Locke utiliza, en determinados pasajes, el término esencia real (véase Ayers, 1975, 14-16; Bolton, 1976, 503; Leyden, 1969, 227), es claro que la crítica que se realiza a la idea de sustancia es en términos de esencia nominal como idea vaga e inadecuada, es decir, como universal abstracto (véase Simmons, 1992, 20-21).

Ahora bien, en el caso de las máximas morales, al igual que en el de las afirmaciones de las matemáticas, la esencia real y la nominal coinciden, en la medida en que, al carecer de correlato empírico, lo que las hace ser lo que son, sólo puede surgir de la definición de ideas simples y su derivación lógica y formal (véase

nuestras concepciones y aprehensiones como la idea de una substancia espiritual o espíritu" (Essay, II, XXIII, \$5, véase también II, XXIII, \$23).

36 El término en el original es "pictures". 
Essay, IV, III, $\$ \$ 1,18$, IV, IV, $\$ 6, I V$, V, $\$ 11$, IV, XII, \$8-9, I, IV, $\$ \$ 1$, 4). En este punto, ante la falta de demostración formal de las máximas morales que hemos visto Locke presenta en su obra, incluso aceptando la prueba de la procedencia divina, persiste la objeción que supone la crítica a la idea de sustancia por su vaguedad e indeterminación.

En este sentido, podemos decir que la falta de definición precisa resulta incluso más problemática en el caso de la ley de la naturaleza que en el de un objeto empírico, dado que no cuenta con el aporte de los sentidos sino que posee una existencia meramente formal. Hemos visto en el primer apartado que en el caso de las matemáticas y de la moral la definición y demostración formal constituye tanto la esencia nominal como la esencia real del objeto. Hemos visto también que tales objetos pueden tener realidad sin existencia empírica, pero ello exige una definición de sus ideas simples y una demostración de su derivación en ideas complejas. La realidad de la moral, al igual que la de la matemática, debería surgir de ideas simples que no podrían ser sino de reflexión, puesto que nunca podrían obtenerse por sensación tratándose de máximas de deber. Sin embargo, los contenidos de la ley de la naturaleza que hemos analizado hasta aquí (los diez mandamientos, el ama a tu prójimo como a ti mismo, la auto-preservación del género humano, la prohibición de acumular frutos para que se pudran, el deber de caridad, de trabajar la tierra y de heredar la propiedad a los hijos) constituyen ideas complejas. De este modo, su esencia real se vuelve susceptible a la crítica a las esencias nominales de los objetos del mundo, y ello no sólo por falta de una demostración formal sino por falta de una presentación de las ideas simples en las que se basan.

Ahora bien, para comprender el alcance limitado de esta crítica, es preciso señalar que Locke no rechaza in toto el aporte al conocimiento de la idea de sustancia como esencia nominal. En efecto, la esencia nominal nos dice algo del objeto, aun cuando, por su alto grado de generalidad y abstracción, resulte oscura y confusa (véase Essay, IV, IV, \$11). En el mismo sentido, las formulaciones que hemos considerado hasta aquí de los contenidos de la ley de la naturaleza, poseen cierta utilidad como criterio general para la determinación del derecho positivo. De aquí que esta crítica no resulte externa, ya que el propio Locke reconoce la vaguedad y generalidad de estos mandatos, al plantear tanto la necesidad de interpretación por parte de la razón de los mandatos divinos (véase Essay, IV, XVIII, $\$ \$ 10-11,6$ ), como la necesidad de convertir el derecho natural en derecho positivo. En efecto, en ambos casos, Locke sostiene que no se trata sólo de un problema de aplicación, sino de interpretación de la ley de 
la naturaleza (véase TT, II, $\$ \$ 13,123-128,184$; Essay, IV, XVI, $\$ 12$, IV, XX, $\$ 16$, II, XXXIII, \$18). De modo que esta crítica interna, es decir, asimilable por la propia propuesta política lockeana, debe distinguirse de la crítica externa, en términos de inconsistencia o contradicción, que propone Hall.

El entendimiento humano construye la esencia nominal a partir del material sensible provisto por los órganos externos. En consecuencia, el entendimiento humano se involucra en un vano intento por descubrir cuál es la verdadera esencia de un objeto natural. Si la verdad moral es exacta, esto es, demostrable, y si la verdad moral se basa en la intrínseca naturaleza de la persona humana (a pesar de que Locke sostiene que no podemos conocer la esencia real de los objetos), entonces nosotros nos encontramos acorralados en una contradicción (Hall, 1981, 73-74; véase también Abdo Ferez, 2013, 349).

La objeción que subyace a esta crítica, común a Hall y Leyden, es que la esencia real del hombre debe ser conocida para poder deducir de ella la ley de la naturaleza (véase Leyden, 1965, 52-58). ${ }^{37}$ Sin embargo, como bien destaca Lenz, el hecho de que la ley de la naturaleza y la naturaleza humana se hallen en concordancia necesaria, no supone que el hombre deba conocer la esencia real del hombre para inferir la ley de la naturaleza (de hecho, hemos visto que Locke critica esta deducción hobbesiana) (véase Lenz, 1956, 108; Chumbita, 2015). En efecto, como hemos podido ver en el tercer apartado de este trabajo, y contra la crítica de Hall, la ley de la naturaleza obliga como mandato divino, con independencia del conocimiento que el hombre tenga de su esencia real. La adecuación ontológica entre la ley de la naturaleza y la naturaleza humana se sigue de la creación divina tanto del hombre como de la ley de la naturaleza, pero ello no significa que el conocimiento de la ley de la naturaleza constituya un producto derivado del conocimiento de la esencia humana (véase ELN, I, 116117, IV, 156-157; Simmons, 1992, 26; Aarsleff, 1969, 100; Singh, 1961, 112). Si bien la ley de la naturaleza y el hombre son ambos creaciones divinas, desde el punto de vista epistemológico el hombre no deduce la ley de la naturaleza de la naturaleza humana. La ley de la naturaleza está compuesta por deberes de los hombres para con los hombres, pero, como hemos podido ver, se basan en la

37 De hecho, Leyden plantea que Locke maneja dos fuentes de obligación moral contradictorias como ya hemos mencionado (véase Leyden, 1965, 52-58). Sin embargo, para Locke Dios es la única fuente de obligación moral, véase ELN, VI, 182-185; Essay, II, XXVIII $\$ 8 ;$ TT, II, \$6). Para un tratamiento detallado de esta cuestión véase Chumbita, 2015. 
propiedad divina de la vida humana, no en el conocimiento que el hombre tenga de sí.

\section{Conclusión}

Podemos concluir, por tanto, que la crítica lockeana a la idea de sustancia se aplica al concepto de la ley de la naturaleza. En efecto, a partir de lo considerado en el segundo apartado, podemos señalar que Locke no ofrece una demostración formal para determinar el contenido de la ley de la naturaleza. En este sentido, incluso aceptando la prueba de los principios del cristianismo a partir de la certificación divina de los milagros, ello no logra eliminar el problema que supone el grado de generalidad de sus formulaciones. En el tercer apartado, a partir del estudio de los diferentes contenidos propuestos en el corpus lockeano, se advirtió que éstos no se siguen de un principio unitario, lo cual no implica asumir la crítica radical de Strauss, según la cual la ley de la naturaleza no es más que una construcción subjetiva del hombre, sino las más modestas apreciaciones de Yolton a este respecto. En el cuarto apartado, a partir de la revisión de la crítica a la idea de sustancia en tanto esencia nominal, hemos podido constatar que las diversas formulaciones del contenido de la ley de la naturaleza constituyen ideas complejas, de las que no se presentan sus ideas simples, por lo que no puede explicarse cómo éstas habrían de derivar en aquéllas, en concordancia con las exigencias de la epistemología lockeana. De este modo, podemos concluir que la ley de la naturaleza constituye un universal abstracto debido a su grado de generalidad y falta de sustento en ideas simples. Tal crítica ha sido definida como interna, en la medida en que se corresponde con la necesidad, reconocida por el propio Locke, de interpretación de los mandatos divinos a partir de la razón individual y, en la sociedad política, de su determinación a través del derecho positivo o la voluntad popular. En este sentido, se dio cuenta de su diferencia con respecto a las críticas que, como las de Leyden y Hall, niegan toda utilidad y consistencia a la teoría lockeana de la ley de la naturaleza. De este modo, puede concederse a la clave de lectura de Strauss que la mediación de la interpretación individual de la ley de la naturaleza conduce a determinados conflictos en estado de naturaleza, dado el grado de generalidad y vaguedad de su contenido, especialmente cuando se realiza de modo parcial o interesado. Sin embargo, recuperando la interpretación de Yolton, puede decirse que una consideración exhaustiva de las fuentes lockeanas no permite subestimar la importancia de la teología cristiana tanto en la determinación del contenido de la moral como en su validación epistemológica, razón por la cual la problemática interpretación del contenido de la ley de la naturaleza por parte de los hombres, no necesariamente 
conduce a consecuencias de un estado de naturaleza hobbesiano que Strauss extrae, precisamente en virtud de la importancia ética y epistemológica de la garantía teológica.

\section{Bibliografía}

\section{Primaria}

LOCKE, J. (1824). Works of John Locke in Nine Volumes. London: Rivington.

(1965). Essays on the law of nature. The Latin text with a translation, introduction and notes, together with transcripts of Locke's shorthand in his journal of 1676. Ed. W. Von Leyden. Oxford: Oxford University Press.

(1967). Two Tracts on Government. Tr. Philip Abrams. Cambridge: Cambridge University Press.

(1977). La racionalidad del cristianismo. Tr. Leandro González Puertas. Madrid: Ediciones Paulinas.

(1990). Segundo Tratado sobre el Gobierno Civil. Un ensayo acerca del verdadero origen, alcance y fin del Gobierno Civil. Tr. Carlos Mellizo. Madrid: Tecnos.

(1991). "Examen de la opinión del padre Malebranche de ver todas las cosas en Dios." Obras varias y correspondencia de (y sobre) John Locke. Tr. José Antonio Robles y Carmen Silva. México: Universidad Autónoma Metropolitana: 35-81.

(2002). Political Essays. Ed. Mark Goldie. Cambridge: Cambridge University Press.

(2004). Two Treatises of Government. Tr. Peter Laslett. Cambridge: Cambridge University Press.

(2005). Ensayo sobre el entendimiento humano. Tr. Edmundo O'Gorman. México: Fondo de Cultura Económica.

(2007). La Ley de la Naturaleza. Tr. Carlos Mellizo. Madrid: Tecnos.

(2011). Ensayo sobre la tolerancia y otros escritos sobre ética y obediencia civil.

Tr. Blanca Rodríguez López y Diego Fernández Peychaux. Madrid: Minerva.

\section{Secundaria}

AARSLEFF, H. (1969). "The state of nature and the nature of man in Locke." John Locke: Problems and Perspectives. Ed. YOLTON, J. W. Cambridge: Cambridge University Press: 99-136. 
ABDO FEREZ, C. (2013). "Hombría, colonialismo y castigo en John Locke". Crimen y sí mismo. La conformación del individuo en la temprana modernidad occidental, Buenos Aires, Gorla: 335-451.

ASHCRAFT, R. (1968). "Locke's State of Nature. Historical fact or Moral Fiction?" The American Political Science Review 62.3: 898-915.

(1969). "Faith and knowledge in Locke's philosophy." John Locke: Problems and Perspectives. Ed. YOLTON. J. W. Cambridge: Cambridge University Press: 194-223.

AYERS, M. R. (1975). "The Ideas of Power and Substance in Locke's Philosophy." The Philosophical Quarterly 25. 98: 2-27.

BOLTON, M. B. (1976). "Substrata, and Names of Substances in Locke's Essay." The Philosophical Review 85. 4: 488-513.

CHUMBITA, J. S. (2011). "El desplazamiento en la teoría de la propiedad de John Locke: del criterio de necesidad a la teoría del valor para justificar la colonización inglesa en América", Cuyo: Anuario de filosofia Argentina y Americana 28: 93-120.

(2013a). "Un análisis de las nociones de abundancia y esclavitud para reinterpretar el carácter universal de la teoría de la apropiación de John Locke", Las torres de Lucca 2: 69-83.

(2013b). "La caridad como administración de la pobreza", Identidades, Revista del Instituto de Estudios Sociales y Políticos de la Patagonia 4: 1-21.

(2014a). "Actores sociales y económicos en las propuestas jurídicas y normativas de John Locke", en Anales del Seminario de Historia de la Filosofía 31: 89-105.

(2014b). "Teología política, libertad natural, paz relativa y secularización en el estado de naturaleza descrito por John Locke”, Bajo palabra 9: 191-202.

(2015). "La ley de la naturaleza como mandato divino. Continuidades entre los escritos tempranos y de madurez en la obra de John Locke", Telos. Revista de Estudios Interdisciplinarios en Ciencias Sociales 17.1: 120-150.

(2016a). "La relación entre latitudinarismo, escepticismo, tolerancia y protestantismo en la obra de John Locke", Contrastes. Revista Internacional de Filosofia XXI (en prensa).

(2016b). "La vigencia de la ley en el estado de naturaleza lockeano: libertad, castigo, guerra justa y esclavitud”, Signos flosóficos, México D.F., Departamento de Filosofía de la Universidad Autónoma Metropolitana, 35 (en prensa).

COX, R. (1960). Locke on War and Peace. London: Oxford University Press.

DI BIASE, G. (2013). "The Development of the Concept of Prudentia in Locke's Classifications of Knowledge", Society and Politics 7. 2: 85-125. 
DUNN J. (1969). The political thought of John Locke. An historical account of the argument of the "Two Treatises of government". Cambridge: Cambridge University Press

GAELA ESPERANZA, J. (2006). John Locke and the Natural Law. Yesterday and today: a critical analysis. Extracto de Tesis Doctoral. Facultad Eclesiástica de Filosofía. Pamplona: Universidad de Navarra.

GAROTTI, L. R. (1961). Locke e i suoi problemi, Urbino, Publicazioni dell'Università di Urbino.

GOUGH, J. W., John Locke’s Political Philosophy. Oxford: Oxford University Press, 1964.

HALL, R. D. (1981). John Locke and Natural Human Rights: an Inconsistency between his metaphysicallepistemological positions and his moral/political theories. Michigan: University Microfilms International.

HANCEY, J. O. (1976). "John Locke and the Law of Nature." Political Theory 4: 439454.

HOBBES, T. (1999). Leviatán. Tr. Carlos Mellizo. Buenos Aires: Alianza Editorial.

LENZ, J. W. (1956). "Locke's Essays on the Law of nature." Philosophy and Phenomenological Research, 17: 105-113.

LEYDEN, W. Von (1965). "Introduction". Essays on the law of nature. The Latin text with a translation, introduction and notes, together with transcripts of Locke's shorthand in his journal of 1676. Oxford: Oxford University Press.

SHAPIRO, B. (1968). "Latitudinarianism and Science in Seventeenth-Century England." Past \& Present, 40 (1968): 16-41.

SELIGER, M. (1963). "Locke's Natural Law and the Foundation of Politics." Journal of the History of Ideas, 24 (1963): 337-354.

SIMMONS, A. J. (1992). The lockean theory of rights. Princeton: Princeton University Press.

SINGH, R. (1961). "John Locke and the Theory of Natural Law." Political Studies 9: 105-118.

STRAUSS, L. (1958). "Critical Note: Locke's Doctrine of Natural Law." American Political science Review 52: 490-501.

(1992). Natural Right and History. Chicago: Chicago University Press.

UDI, J. (2012). "Propiedad lockeana, pobreza extrema y caridad", Revista de Estudios Politicos 157: 165-188.

(2014). "El derecho a la caridad: repercusiones de la teología cristiana en la teoría de la propiedad de John Locke", Revista de Filosofía 70: 149-160. 
YOLTON, J. W. (1958). "Locke on the Law of Nature." The philosophical Review 67: 477-498.

John Locke: and the way of ideas. Cambridge: Cambridge University Press, 1968.

WALDRON, J. (2002). God, Locke and Equality: Christian Foundations in Locke's Political Thougth. Cambridge: Cambridge University Press.

(2005). "Response to Critics", The Review of Politics, Vol. 67, N 3 (Summer), 495-513.

Enviado: 15/05/2015

Aceptado: 19/10/2015

Este trabajo se encuentra bajo una licencia de Creative Commons ReconocimientoNoComercial-SinObraDerivada 4.0

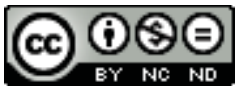

ÉNDOXA: Series Filosóficas, n. 36, 2015, pp. 99-122. UNED, Madrid 
